\title{
Brightness exponent as a function of flash duration and retinal eccentricity
}

\author{
NAOYUKI OSAKA \\ Otemon-Gakuin University, Ibaraki, Osaka 567, Japan
}

\begin{abstract}
The effect of flash duration on the exponent of the brightness power function was investigated in the fovea and 20-deg periphery using a method of direct magnitude estimation. The flash duration employed covered a 5-log-unit range between .001 and $100 \mathrm{sec}$. The results showed that the exponent is clearly time-dependent for both extremes of the duration-that is, very short $(.001$ to $.1 \mathrm{sec}$ ) and prolonged ( 3 to $100 \mathrm{sec}$ ) durations-but not for the medium flash durations between .1 and $3 \mathrm{sec}$. Furthermore, the exponent is a little larger for peripheral viewing than for foveal viewing except for the durations below approximately $.03 \mathrm{sec}$. The systematic change of the exponent as a function of duration is discussed in terms of retinal and postretinal intensity coding functions.
\end{abstract}

At suprathreshold luminances, perceived brightness has been known to be time-dependent for transient-state flash durations less than critical duration, within which Bloch's law holds, whereas it has been known to be relatively time-independent for steady-state flash durations well above critical duration. However, for prolonged flash durations-say, more than several seconds-perceived brightness evoked by a relatively lower flash luminance gradually decreases due to the Troxler fading effect (Clarke, 1960). Furthermore, for prolonged durations, the Troxler fading effect has been known to occur in great magnitude when a target is viewed peripherally. However, for transient durations, a target appears brighter for peripheral viewing than for foveal viewing (Marks, 1971; Osaka, 1977, 1980).

In this context, any perceived brightness is timeand eccentricity-dependent, although "steady-state" flash is employed. Studies investigating an intensity coding mechanism on vertebrate receptors (Boynton \& Whitten, 1970; Mansfield, 1974) and psychophysical brightness power functions (Mansfield, 1973; Osaka, 1977, 1978, 1980; J. C. Stevens \& Hall, 1966) suggest that flash signals are nearly linear with brief flashes within Bloch region and nonlinear with long flashes. This implies that the size of the exponent of the brightness power function decreases from approximately 1.0 (linear coding) to .33 (cubic root coding) as flash duration increases from 1 to $1,000 \mathrm{msec}$ (Osaka, 1977). However, for prolonged durations, it is likely that the exponent of the brightness power function changes due to long-term brightness adaptation and the Troxler fading effect. Little study has been reported on the global exponent changes

This research was supported in part by Grant 401059 from the Japan Ministry of Education. as a function of eccentricity and flash durations with extended range.

The aim of the present paper is to provide and test a global model on the time-dependent brightness exponent in the fovea and periphery using extended flash durations covering the range between $1 \mathrm{msec}$ and 100 sec-a 5-log-units range.

\section{Model on Time-Dependent Brightness Function}

Figure 1 shows seven possible forms of the timedependent brightness functions. Each panel indicates different time-dependent brightness functions for three different suprathreshold luminance levels as a parameter. It should be noted that each brightness vs. duration curve is plotted in a $\log -\log$ plot. In panel $\mathbf{A}$, brightness is independent of duration; thus, brightness is determined solely in terms of flash luminance, whereas brightness decreases (panel B) and increases (panel C) as duration increases. However,

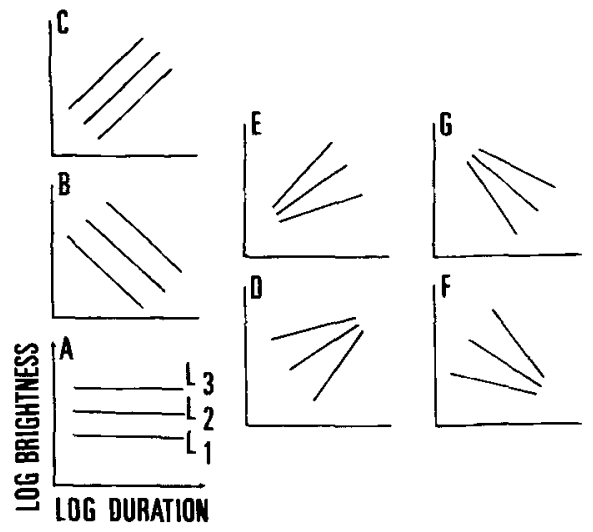

Figure 1. Possible time-dependent brightness functions. An equal-Iuminance contours; brightness vs. duration curves in a loglog plot. The parameter is luminance. 
the ratio of brightness change is independent of duration. These three panels $(A, B$, and $C)$ suggest that the brightness exponent is independent of flash duration as long as the three constant luminance curves parallel each other. If the luminance value that is required to produce constant brightness is plotted as a function of duration in a log-log plot, the resulting function, except for that of panel $A$, has an opposite form. The ratio of brightness change to luminance is clearly dependent on the flash duration in a complex manner, as shown in panels $D$ through $G$ in Figure 1. This results in a change of flash exponent as a function of flash duration. As the ratio increases, the exponent increases, and vice versa.

\section{EXPERIMENT 1}

\section{Method}

Observers. Six persons (five male and one female), who had normal vision, served as observers. All observers had had extensive experience in magnitude estimation judgments.

Apparatus. A microcomputer-based (Commodore PET 2001-8 type) setup was used for experimental control. The pulse motor driver, Glow tube driver, and buffer driver were under program control via an eight-bit bidirectional parallel user port within the PET (Osaka, 1979a). A Sylvania Glow Modulator tube (Type R1131C) with rise and fall times shorter than $.01 \mathrm{msec}$ was mounted in the lamp housing as a light source. The luminance of the flash was changed by means of Kodak Wratten 96 neutral density filters, which were mounted in the circular wheel connected to the pulse motor (see Osaka, 1979a, for details). Four luminances were used, covering the range between .25 and $250 \mathrm{~cd} / \mathrm{m}^{2}$ in steps of $1 \mathrm{log}$ unit. The luminance was measured using a digital luminance meter (Minolta 1-deg digital). The size of the circular target was set at a .75 -deg visual angle. Two retinal loci along the horizontal meridian of the right eye were explored: the fovea and a 20-deg temporal field. The order of selection of the neutral density filter (luminance control) as well as the flash duration were controlled by a string of random pulses generated by the PET. Using the PET as a square pulse generator, 11 flash durations were employed: $.001, .005, .01$, $.05, .1, .5,1,5,10,50$, and $100 \mathrm{sec}$. The fixation point was a just visible light-emitting diode ( $650 \mathrm{~nm}$ peak wavelength).

Procedure. After $15 \mathrm{~min}$ of dark-adaptation, the observer's head was immobilized by a chin- and forehead-support that set his right eye at $30-\mathrm{cm}$ viewing distance. The test flashes, varying in duration and luminance, were presented in random order at each retinal location. Using the method of direct magnitude estimation, each observer judged each flash four times, thus performing a total of 11 by 4 by 2 by 4 (i.e., combinations of duration, luminance, locus, and trial, respectively). The reference flash was a foveally (left eye) presented flash of $2.5 \mathrm{~cd} / \mathrm{m}^{2}$ (1-sec duration), the brightness of which was assigned the reference value of " 100 "; it was presented to the left eye $1 \mathrm{sec}$ after the offset of the test flash presented to the right eye. The observer viewed the test flash with the right eye, during which the left eye was occluded with an electronic shutter. The left eye shutter was opened (for $2 \mathrm{sec}) 1 \mathrm{sec}$ after the offset of the test flash, during which time the right eye was occluded with the shutter. The shutter operation was controlled by the PET.

The observer was asked to give the test flash brightness any number that appeared to him most appropriate to stand for the perceived brightness in proportion to the brightness of the reference flash, ignoring as much as possible any differences in perceived flash duration. The observer was asked to judge the brightness just before the offset of the test flash for longer durations. Each brightness estimate datum obtained was stored via PET's keyboard (by the experimenter), processed, and printed (CBM
3022 printer) with their stimulus parameters in real-time. The time between the offset and onset of the test flash was randomized from 10 to $15 \mathrm{sec}$ by the random function of the PET (Osaka, 1979b).

\section{Results and Discussion}

The log geometric mean of 24 estimates for each luminance level is plotted against log flash duration in Figures 2 and 3, which show the plot of foveal and of peripheral viewing, respectively. The lines in the figures were fitted by the eye. In foveal viewing, perceived brightness grew as a function of increasing flash duration up to approximately $50-100 \mathrm{msec}$, beyond which the brightness was determined solely in terms of flash luminance up to approximately $10 \mathrm{sec}$. However, interestingly enough, the brightness gradually tended to decrease as flash duration was prolonged over $10 \mathrm{sec}$ up to some asymptotic level. In a similar way, brightness grew, leveled off, and decreased as duration increased in peripheral viewing, except that the rate of brightness decrease appeared somehow more rapid and fluctuated more

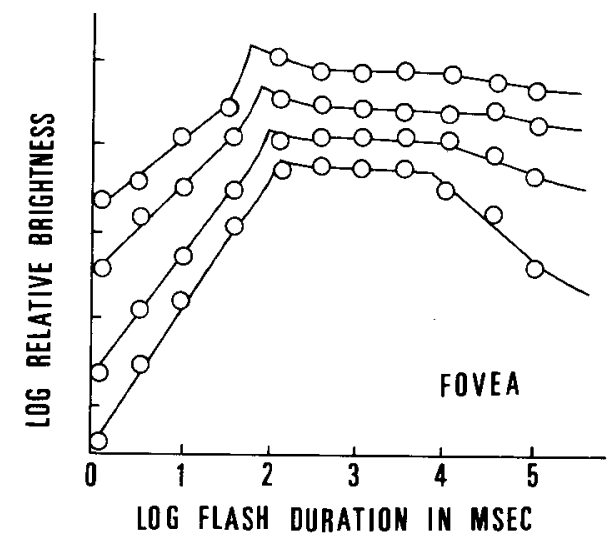

Figure 2. Log relative brightness as a function of $\log$ duration in the fovea. The parameter is luminance. Brightness grows, levels off, and declines as flash duration increases.

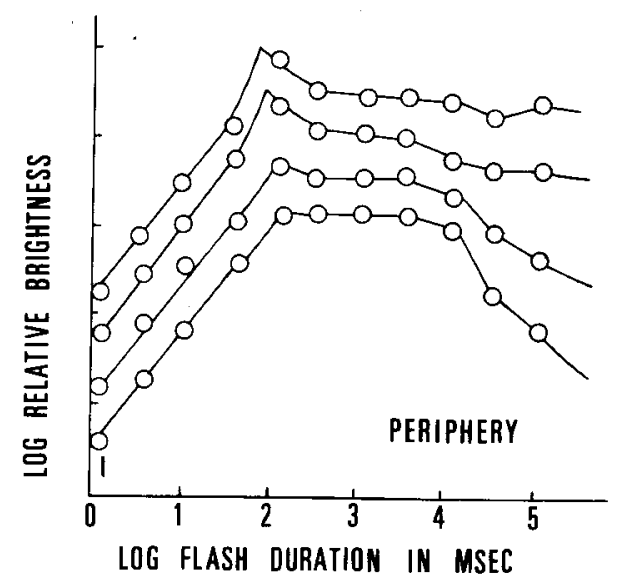

Figure 3. $\mathrm{Log}$ relative brightness as a function of $\log$ duration in the 20-deg periphery. The parameter is luminance. Brightness decreases more rapidly than foveal viewing for prolonged durations. 
than foveal viewing for prolonged and lower luminance flashes. This fluctuation of brightness is probably due to Troxler fading. A target always appeared about $.3 \mathrm{log}$ units brighter for peripheral viewing than for foveal viewing when 1-sec flash was employed. This effect has been found by previous studies (Marks, 1971; Osaka, 1975, 1977, 1980). The results are generally in good agreement with the data by other investigators (Aiba \& S. S. Stevens, 1964; Osaka, 1977; Raab, 1962; J. C. Stevens \& Hall, 1966) insofar as the range of the duration was between 1 and $1,000 \mathrm{msec}$. An overall shape of the brightness function indicates that brightness increased (see panel $D$ for fovea and panel $C$ for periphery), leveled off (panel $A$ for both), and decreased (panel $G$ for both) as duration increased (cf. panels in Figure 1).

The ratio of the brightness change, as revealed by the size of the exponent, is shown in Figure 4. The exponent was estimated by the method of least squares. Figure 4 indicates that the brightness exponent has a deep- and shallow-U-shaped function when it is plotted against duration for the fovea and periphery, respectively. The exponent decreased (panel D), leveled off (panel A), and increased (panel G) for the fovea but remained nearly constant (panels $C$ and $A$ ) and increased (panel G) for the periphery (cf. corresponding brightness functions in Figure 1).

For $1 \mathrm{msec}$ duration in the fovea, brightness grew faster than any other durations as luminance increased: Brightness is a nearly linear function of luminance since the exponent is close to 1.0. Nevertheless, brightness remains a square root function of luminance in the 20-deg periphery of the same condition. This implies that the intensity coding mechanism is nearly linear for the brief flash durations in

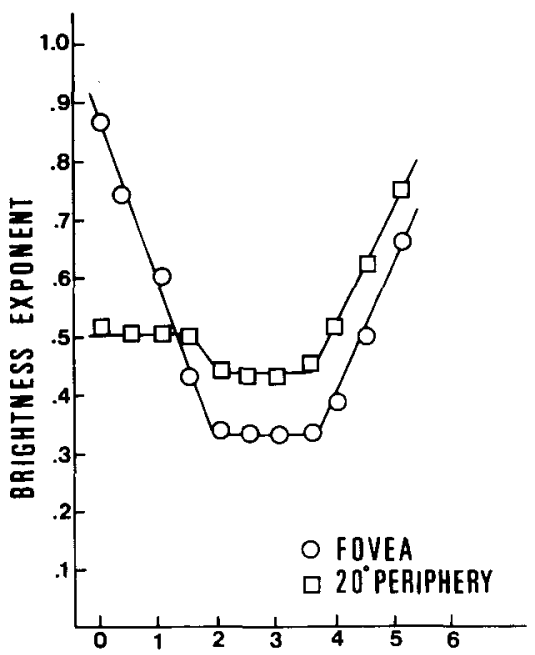

LOG FLASH DURATION IN MSEC

Figure 4. Brightness exponent as a function of $\log$ flash duration. Exponent decreases, levels off, and grows as flash duration increases. Note the deep- and shallow-U-shaped function for the fovea and periphery, respectively. the foveal system, while it is almost a square root $(1 / 2)$ coding for the transient flash durations in the peripheral system. For the intermediate durations, however, intensity coding is nearly cube root $(1 / 3)$ and square root $(1 / 2)$ for the fovea and periphery, respectively. It should be noted that cube root coding is valid for the fovea but not for the periphery. Moreover, for prolonged durations, the exponent again increases as duration increased due to rapid decrease of brightness for the lower luminance target for both retinal locations.

\section{EXPERIMENT 2}

A time-dependent decrease in the sensitivity of a visual system is called long-term visual adaptation. It is likely that the brightness fluctuation observed for prolonged durations is probably due to a Troxler phenomenon, a special aspect of adaptation. To test the hypothesis, the size of the target was changed, since the Troxler fading effect has been known to be sensitive to the target size (Clarke, 1960).

\section{Method}

Observer. All of the observers were the same as those who had participated in Experiment 1.

Apparatus. The apparatus was the same as that used in Experiment 1 , with the following differences. Three stimulus sizes were employed: 80, 45, and $25 \mathrm{~min}$ visual angle. Furthermore, 11 flash durations were employed: $1,2,4,6,8,10,20,30,40,50$, and $60 \mathrm{sec}$.

Procedure. The procedure used was the same as that employed in Experiment 1.

\section{Results and Discussion}

The log relative brightness is plotted as a function of $\log$ duration in Figure 5. The parameter is luminance, and lines were fitted by the eye. Any target was perceived as about .2 to $.4 \mathrm{log}$ units brighter for peripheral viewing than for foveal viewing.

As Figure 5 shows, perceived brightness again appears to decrease with prolonged flash duration, although the trend is relatively smaller for the higher luminance targets, implying that luminance must be increased to maintain equal brightness as duration increases. Moreover, the results show that the brightness is clearly dependent on stimulus size: As stimulus size decreases, brightness decreases more rapidly as a function of increasing duration. It was also found that this trend is evident for peripheral viewing, which implies that the Troxler fading effect is more dominant in the periphery than in the fovea.

The slope of the dotted line drawn through the points at which the brightness is going to decrease can be roughly estimated by the angle $\theta$ : As target size decreases, $\theta$ slightly decreases. Furthermore, the magnitude of $\theta$ is always larger for foveal viewing than for peripheral viewing for each stimulus size condition.

In the peripheral viewing, brightness again fluc- 

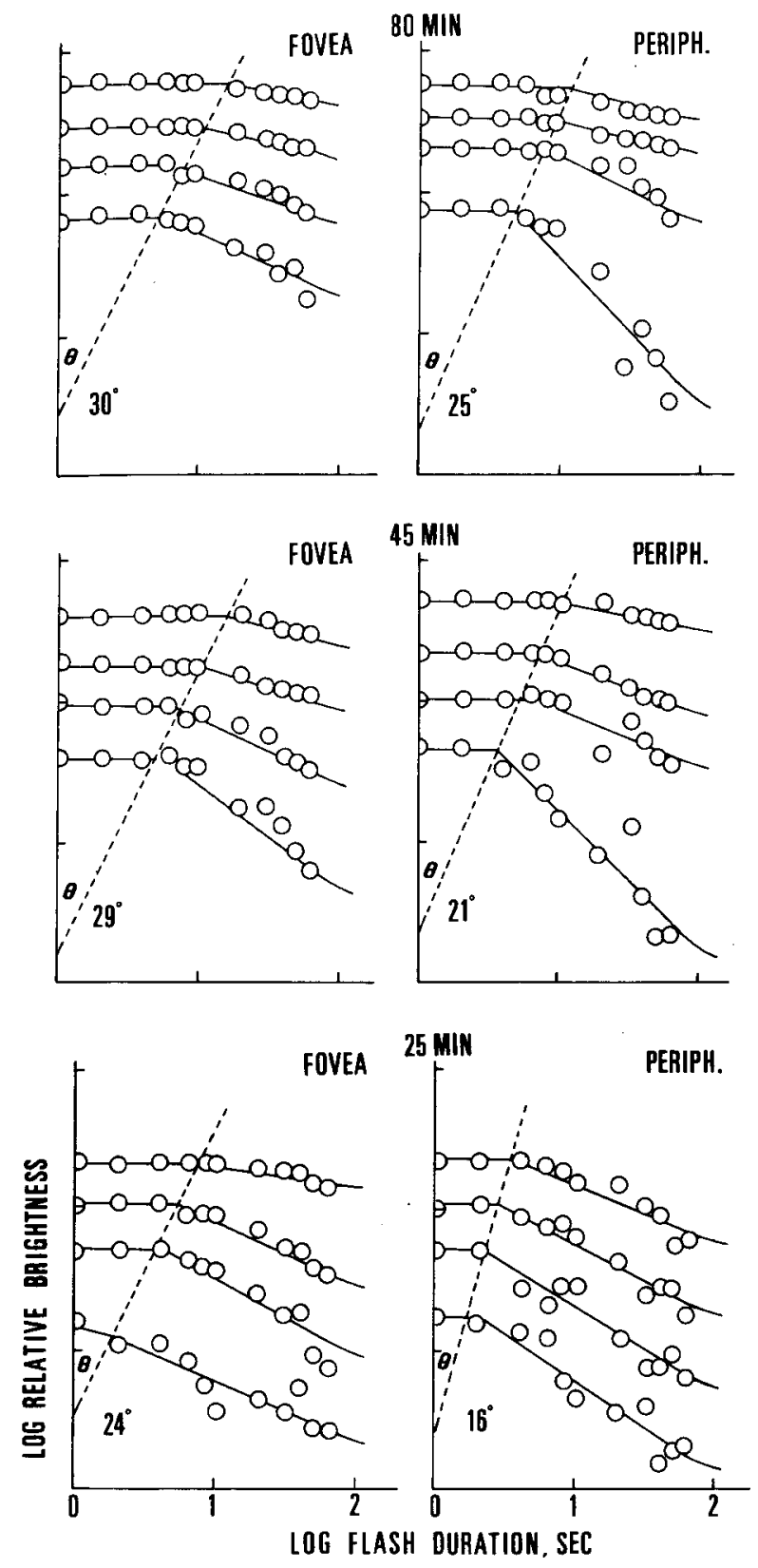

Figure 5. Troxler fading function; $\log$ relative brightness as a function of log prolonged duration in the fovea and periphery. Each pair of panels shows different target size. The parameter is luminance. The angle $\theta$ indicates the degree of intensity-dependent initial brightness change for each luminance level.

tuated for prolonged durations (from 10 up to $60 \mathrm{sec}$ ) when the lowest luminance target was employed: Some observers reported that the target faded out (i.e., "zero" brightness) for a few seconds and then suddenly reappeared. This can be explained in terms of voluntary eye movements and related transient stabilization of the retinal image (Riggs, Armington, \& Ratliff, 1954).

Clarke (1960) found that perceived brightness rapidly decreases after a few seconds of an initial de- lay and that Troxler fading becomes marked with increase of eccentricity. The results of the present study show that the duration of the initial delay ranges from 2 to $10 \mathrm{sec}$, whereas Clarke (1960) found the delay ranges from 1 to $5 \mathrm{sec}$. At $20 \mathrm{deg}$ eccentricity (upper meridian of the field), the stimulus size up to 2 deg faded and disappeared (Clarke, 1960), while at $20 \mathrm{deg}$ eccentricity (temporal meridian of the field), any stimulus size up to $1.3 \mathrm{deg}$ did not completely disappear but fluctuated in brightness in the present experiment. The differences can partly be explained in terms of differences in methodology: The test stimulus was presented for $.5 \mathrm{sec}$ to a dark-adapted periphery, about every $5 \mathrm{sec}$, and its brightness was adjusted to match that of the foveal stimulus as seen by the light-adapted fovea (Clarke, 1960), whereas, in the present experiment, the test flash was presented continuously and the brightness was estimated by the method of magnitude estimation on the basis of 1-sec flash brightness presented to the dark-adapted left eye.

The results show that the time course of the brightness adaptation is markedly different from another when different luminances are employed. Figure 6 shows that as the flash duration increases, brightness exponent increases from .30 to .70 due to adaptation as well as to Troxler fading, reconfirming the results obtained in the Experiment 1 (cf. panel $G$ in Figures 1 and 4). It should be noted that the peripheral

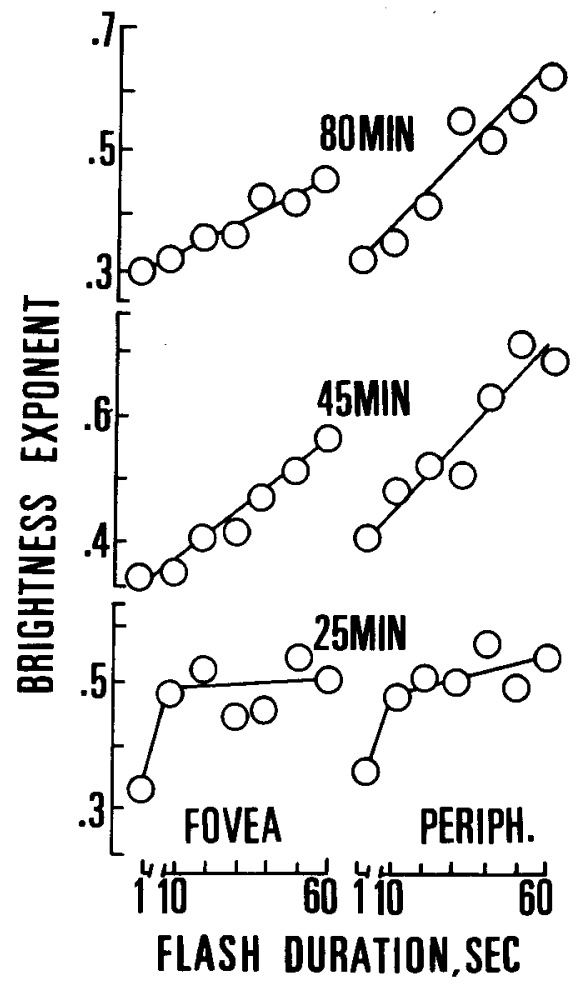

Figure 6. Brightness exponent of the Troxler fading function as a function of flash duration for different target size in the fovea and periphery. 
exponents are also larger than that of the fovea at any durations except for the minimum target size. A similar study, investigating the time course of brightness adaptation in the fovea, has been published (Geldard, 1928a, 1928b; Wallace, 1937). Using a direct brightness matching technique, Geldard measured a "fatigue curve," up to about $120 \mathrm{sec}$, for brightness following prolonged duration of a flash for three intensity levels in the fovea: The results indicate that the time course of brightness change varies with flash intensity. Marks (1974, pp. 115-116) transformed Geldard's original data by applying the cubic root power law and found that the greater the initial brightness, the faster its decline. This disagrees with the present results, which state that the greater the initial brightness, the slower its fading. The present results for higher luminance flash are rather likely to agree with the study by Craik (1940), in which he found that the asymptotic brightness is consistent, regardless of flash luminance, when flash intensity is very high.

\section{GENERAL DISCUSSION}

We have just outlined how brightness changes with time of flash duration over a 5-log-unit range of duration. The results of Experiments 1 and 2 show that the exponent of the psychophysical brightness power function in general involves time-dependent characteristics: From the results of the experiment, it should be concluded that the exponent is clearly timedependent for both extremes of the flash durationthat is, very short $(.001$ to $.1 \mathrm{sec})$ and prolonged ( 3 to $100 \mathrm{sec}$ ) durations-but not for the medium flash durations $(.1$ to $3 \mathrm{sec})$. Furthermore, the exponent is a little larger for peripheral viewing than for foveal viewing, except for the durations below approximately $30 \mathrm{msec}$, at which the sizes interchange with each other. Consider the exponents obtained with a flash of intermediate durations from .1 sec to several seconds (see Figure 4). The exponent is independent of duration, implying that the nature of the intensity coding functions has the same nonlinear characteristics and is practically constant regardless of flash duration.

The present findings indicate that an intensity coding mechanism approaches linearity for both extremes of flash duration: As flash duration decreases, intensity coding becomes nearly linear due to retinal transduction processes (Mansfield, 1974), while, as duration is prolonged, intensity coding becomes quasi-linear due to postretinal adaptation processes (Craik, 1940; Pitt, 1939) that involve Troxler fading (Clarke \& Belcher, 1962). Thus, it may be reasonable to suggest that both retinal and postretinal processes are equilibrated with respect to each other at the intermediate flash durations, in which a "steady-state" flash provides a well-known and stable cubic root power function, as established by S. S. Stevens.

\section{REFERENCES}

Aibn, T. S., \& Stevens, S. S. Relation of brightness to duration and luminance under light- and dark-adaptation. Vision $R e$ search, 1964, 4, 391-401.

Boynton, R. M., \& WhitTen, D. N. Visual adaptation in monkey cones: Recording late receptor potentials. Science, 1970, 170, 1423-1426.

Clarke, F. J. J. A study of Troxler's effect. Optica Acta, 1960, 7, 219-236.

Clarke, F. J. J., \& Belcher, S. J. On the localization of Troxler's effect in the visual pathway. Vision Research, 1962, 2, 53-68.

CraIK, K. J. W. The effect of adaptation on subjective brightness. Proceedings of the Royal Society (London), 1940, B-128, 232-247.

GeldARD, F. A. The measurement of retinal fatigue to achromatic stimulation-I. Journal of General Psychology, 1928, 1, 123135. (a)

GeLDARD, F. A. The measurement of retinal fatigue to achromatic stimulation-II. Journal of General Psychology, 1928, 1, 578590. (b)

Mansfield, R. J. W. Brightness function: Effect of area and duration. Journal of the Optical Society of America, 1973, 63, 913-920.

Mansfield, R. J. W. Measurement, invariance, and psychophysics. In H. R. Moskowitz, B. Scharf, \& J. C. Stevens (Eds.), Sensation and measurement. Dordrecht: Reidel, 1974.

Marks, L. E. Brightness and retinal locus: Effects of target size and spectral composition. Perception \& Psychophysics, 1971, 9, 26-30.

Marks, L. E. Sensory processes: The new psychophysics. New York: Academic Press, 1974.

OsakA, N. Target size and luminance in apparent brightness of the peripheral visual field. Perceptual and Motor Skills, 1975, 41, 49-50.

Osaka, N. Perceived brightness as a function of flash duration in the peripheral visual field. Perception \& Psychophysics, 1977, 22, 63-69.

Osaka, N. Brightness exponent for the periphery in the Bloch region. Journal of the Optical Society of America, 1978, 68, 140-142.

OsAKA, N. A microprocessor-based real-time BASIC laboratory: A pulse motor controlled visual stimulator. Behavior Research Methods \& Instrumentation, 1979, 11, 549-552. (a)

OsakA, N. A microprocessor-based real-time BASIC random interstimulus interval generator with different probability density functions. Behavior Research Methods \& Instrumentation, 1979, 11, 581-584. (b)

OsAKA, N. Brightness exponent as a function of retinal eccentricity in the peripheral visual field. Perception \& Psychophysics, 1980, 27, 519-523.

PITT, F. H. G. The effect of adaptation and contrast on apparent brightness. Proceedings of the Physical Society (London), 1939, 51, 817-830.

RAAB, D. Magnitude estimation of brightness of brief foveal stimuli. Science, 1962, 135, 42-43.

Riggs, L. A., Armington, J. C., \& Ratliff, F. Motions of the retinal image during fixation. Journal of the Optical Society of America, 1954, 44, 315-321.

Stevens, J. C., \& HalL, J. W. Brightness and loudness as functions of stimulus duration. Perception \& Psychophysics, 1966, 1, 319-327.

Wallace, S. R., Jr. Studies in binocular interdependence: $\mathbf{I}$. Binocular relations in macular adaptation. Journal of General Psychology, 1937, 17, 307-322.

(Manuscript received August 25, 1980; revision accepted for publication May 22, 1981.) 